\title{
On the Calculation of Quasi-Bound States and Their Impact on Direct Tunneling in CMOS Devices
}

\author{
A. Gehring and S. Selberherr \\ Institute for Microelectronics, TU Vienna \\ Gußhausstraße 27-29, A-1040 Wien, Austria \\ Email: Gehring@iue.tuwien.ac.at
}

\begin{abstract}
We study the calculation of quasi-bound states in nMOS inversion channels and their impact on direct tunneling currents through the dielectric layer. For typical device parameters, the gate leakage in inversion is dominated by this tunneling component. However, a strong inaccuracy arises, if the eigenvalues of the closed system are used for the quasi-bound state tunneling current. We propose simple correction functions to the closed-boundary eigenvalues calculated by the triangular approximation which allows to account for both, continuum and quasi-bound state tunneling in an efficient manner.
\end{abstract}

\section{Introduction}

The modeling of gate current due to direct tunneling through the gate dielectric layer of CMOS devices has been of increasing interest in recent years. There is strong evidence that under inversion, tunneling happens via quasi-bound states (QBS) in the inverted layer, while under depletion and accumulation, the established Tsu-Esaki formula, which is based on a continuum of states, must be used [1]. Implementations in common device simulator packages still rely on the Tsu-Esaki formula in both cases, which may be due to the cumbersome numerical procedures associated with the QBS approach. However, no comparisons between the continuum and QBS approaches have yet been reported. We study the applicability of both models for nMOS devices with a doping in the range of $1 \times 10^{18} \mathrm{~cm}^{-3}$ up to $9 \times 10^{18} \mathrm{~cm}^{-3}$ and gate dielectric thicknesses of $1.0 \mathrm{~nm}$ up to $3.0 \mathrm{~nm}$.

\section{Tunneling from Continuum versus Quasi-Bound States}

Calculation of tunneling currents is traditionally based on the assumption of a threedimensional continuum of states at both sides of the dielectric and the conservation of parallel momentum. Then, the tunneling current can be described by the Tsu-Esaki formula [2]

$J_{3 \mathrm{D}}=\frac{4 \pi \mathrm{q} m_{3 \mathrm{D}}}{h^{3}} \int_{\mathcal{E}_{\min , 1}}^{\mathcal{E}_{\max }} T C\left(\mathcal{E}_{x}, m_{\text {diel }}\right) N\left(\mathcal{E}_{x}\right) \mathrm{d} \mathcal{E}_{x}$,

where $T C\left(\mathcal{E}_{x}, m_{\text {diel }}\right)$ is the transmission coefficient and $N\left(\mathcal{E}_{x}\right)$ the supply function. Two electron masses enter this equation: the density-of-states mass in the plane parallel to the interface $m_{3 \mathrm{D}}=2 m_{\mathrm{t}}^{*}+4 \sqrt{m_{\mathrm{t}}^{*} m_{1}^{*}}$, which, for (100) silicon with $m_{1}^{*}=0.92 m_{0}$ 

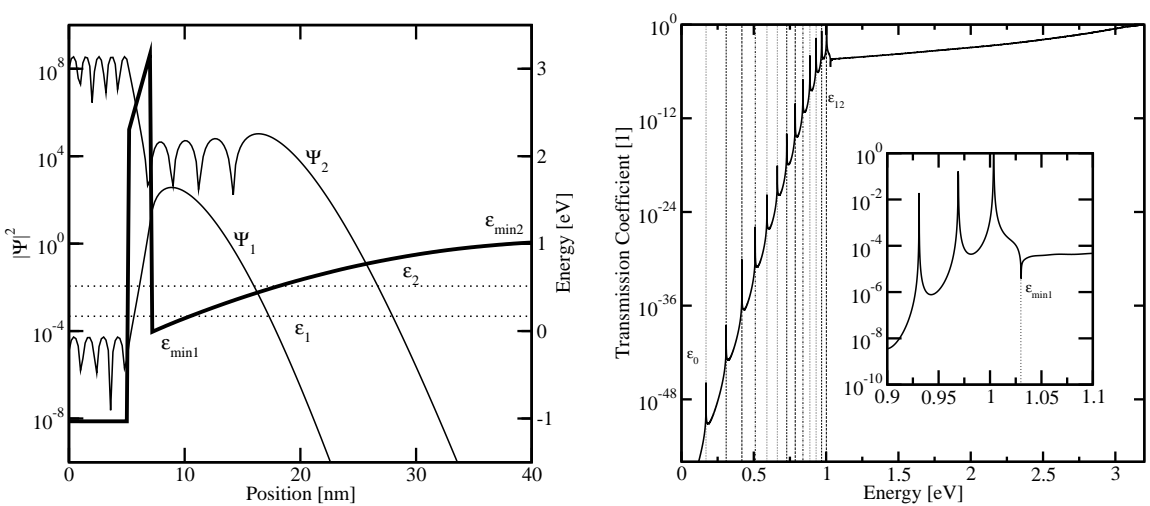

Figure 1: Conduction band profi le of an nMOS Figure 2: Transmission coeffi cient of the left with a doping of $10^{18} \mathrm{~cm}^{-3}$ and $t_{\text {diel }}=2 \mathrm{~nm}$ at structure. For $\mathcal{E}<\mathcal{E}_{\min , 1}$ the calculation $1 \mathrm{~V}$ bias. Calculation of the eigenvalues with effi ciently yields the quasi-bound state energy closed boundary conditions results in spurious levels which perfectly agree with their closedQBS wave functions. boundary pendants.

and $m_{\mathrm{t}}^{*}=0.19 m_{0}$ equals $2.052 m_{0}$, and the electron mass in the dielectric $m_{\text {diel }}$, which is commonly used as fit parameter [3].

However, in the channel of inverted MOS devices, the strong electric field leads to quantum confinement and the assumption of continuum tunneling is no more justified. If assumed that the wave function does not penetrate into the gate, discrete energy levels can be identified. However, it cannot be assumed that electrons tunnel from these energies, since for their calculation zero wave function penetration was postulated. This leads to a paradox which was addressed by MAGNUS and SCHOENMAKER [1]: How can a bound state which has vanishing current density, lead to tunneling current? Taking a closer look at the conduction band edge of an nMOS in inversion (see Fig. 1) reveals that only quasi-bound states lead to tunneling current [4]. The symbols $\mathcal{E}_{1}$ and $\Psi_{1}$ denote the energy level and the wave function of a quasi-bound state. In contrast, bound states are formed at energies for which the wave function decays to zero at both sides of the dielectric and are therefore not encountered in typical nMOS devices.

The QBS tunneling current is proportional to $\sum n_{i} / \tau_{i}$ where $n_{i}$ and $\tau_{i}$ denote the carrier concentration and the life time of the QBS with index $i$, respectively. Several methods have been proposed to calculate the quasi-bound states and their respective life times [5]. One possibility is to calculate the closed-boundary eigenvalues of the whole structure shown in Fig. 1 with zero boundary conditions. With this method, however, spurious states arise ( $\mathcal{E}_{2}$ and $\Psi_{2}$ in Fig. 1$)$ which are due to the artificial boundary conditions. These spurious states must be removed after the calculation [6]. A more efficient approach is based on an evaluation of the transmission coefficient resonances [7]. We used a recursive transmitting-boundary method which is more stable and efficient as the commonly applied transfer-matrix methods [8]. The two approaches are compared in Fig. 2, where the location of the closed-boundary eigenvalues is indicated by lines. Perfect agreement with the more cumbersome closed-boundary approach is achieved. 

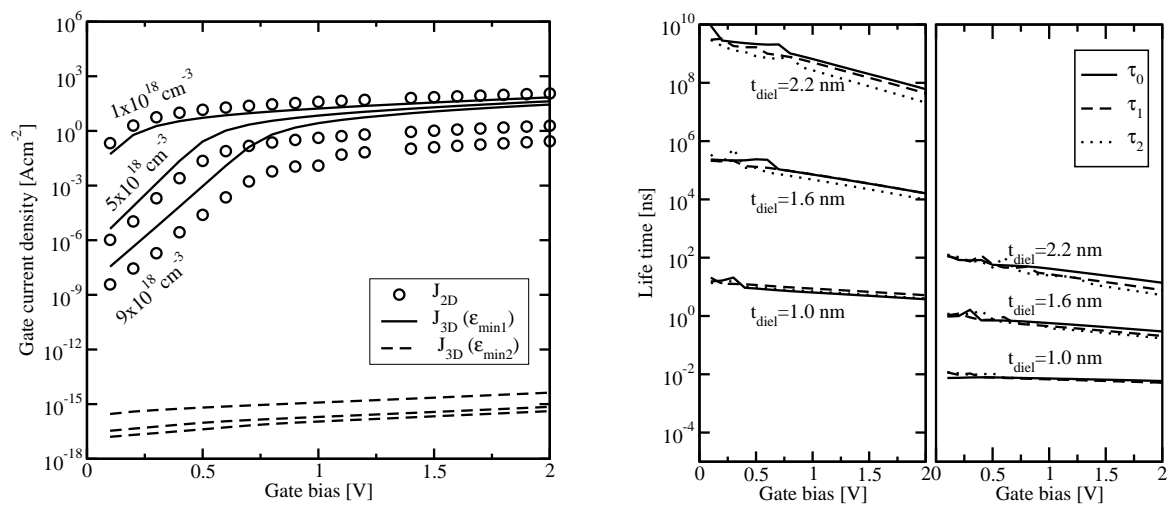

Figure 3: Current density for different bulk Figure 4: Lateral (left) and transversal (right) doping at a dielectric thickness of $1.4 \mathrm{~nm}$. The life times as a function of the gate bias for Tsu-Esaki formula fails to reproduce the QBS $N_{\mathrm{A}}=5 \times 10^{18} \mathrm{~cm}^{-3}$ and different dielectric tunneling. thicknesses.

To account for tunneling current from both, continuum and quasi-bound states, (1) must be replaced by

$$
\begin{aligned}
J=J_{2 \mathrm{D}}+J_{3 \mathrm{D}} & =\frac{\mathrm{k}_{\mathrm{B}} T \mathrm{q}}{\pi \hbar^{2}} \sum_{i, \nu} \frac{g_{\nu} m_{\|}}{\tau_{\nu}\left(\mathcal{E}_{\nu, i}\left(m_{\mathrm{q}}\right)\right)} \ln \left(1+\exp \left(\frac{\mathcal{E}_{\mathrm{F}}-\mathcal{E}_{\nu, i}}{\mathrm{k}_{\mathrm{B}} T}\right)\right) \\
& +\frac{4 \pi \mathrm{q} m_{3 \mathrm{D}}}{h^{3}} \int_{\mathcal{E}_{\text {min }, 2}}^{\mathcal{E}_{\max }} T C\left(\mathcal{E}_{x}, m_{\text {diel }}\right) N\left(\mathcal{E}_{x}\right) \mathrm{d} \mathcal{E}_{x},
\end{aligned}
$$

where the symbols $g_{\nu}, m_{\|}$, and $m_{\mathrm{q}}$ denote the valley degeneracy, parallel, and quantization masses $\left(g=2: m_{\|}=m_{\mathrm{t}}, m_{\mathrm{q}}=m_{\mathrm{l}}\right.$ and $\left.g=4: m_{\|}=\sqrt{m_{\mathrm{l}} m_{\mathrm{t}}}, m_{\mathrm{q}}=m_{\mathrm{t}}\right)$, $\tau_{\nu}\left(\mathcal{E}_{\nu, i}\right)$ is the life time of the quasi-bound state $\mathcal{E}_{\nu, i}$, and the integration in the Tsu-Esaki formula starts from $\mathcal{E}_{\min , 2}$ as indicated in Fig. 1. In Fig. 3 the different current components are compared for example devices. Setting the lower integration level to $\mathcal{E}_{\text {min,2 }}$ shows that for cold-electron tunneling, QBS tunneling is the dominant mechanism. Using $\mathcal{E}_{\min , 1}$ as lower integration level makes the calibration for different substrate doping necessary, therefore the QBS component cannot be neglected. In recent works the 3D tunneling current is often neglected and only QBS tunneling is taken into account. This, however, is also problematic in the case of hot-carrier tunneling [9] or tunneling from accumulation layers where the continuum states can be occupied strongly. It is therefore mandatory to account for both, QBS and continuum tunneling.

The life times $\tau_{\nu}\left(\mathcal{E}_{\nu, i}\right)$ can be calculated from the resonance widths of the transmission peaks in Fig. 2. However, it was shown that the quasi-classical approach

$\tau_{\nu}\left(\mathcal{E}_{\nu, i}\right)=\int_{0}^{x} \frac{\left(2 m_{\nu} /\left(\mathcal{E}_{\mathrm{c}}(\xi)-\mathcal{E}_{\nu, i}\right)\right)^{1 / 2}}{T C\left(\mathcal{E}_{\nu, i}\right)} \mathrm{d} \xi$

with $\mathcal{E}_{\mathrm{c}}(x)=\mathcal{E}_{\nu, i}$, accurately reproduces the results of the more sophisticated calculation procedures [10]. The life times of longitudinal and transversal eigenvalues are shown in Fig. 4 as a function of the gate bias. 

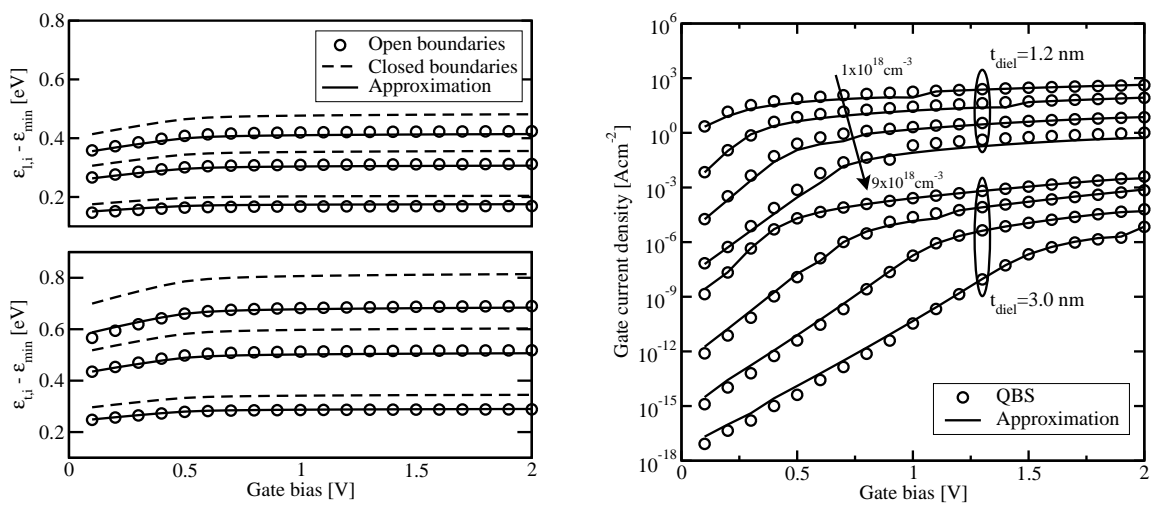

Figure 5: Open- and closed-boundary eigenvalues as a function of the gate bias for $N_{\mathrm{A}}=$ $5 \times 10^{18} \mathrm{~cm}^{-3}$ and $t_{\text {diel }}=1.4 \mathrm{~nm}$, compared to the simple approximation.

Figure 6: Comparison of the full QBS approach (circles) with the approximation (lines) for different substrate doping and dielectric thicknesses.

Still, the detection of the transmission resonances to get the open-boundary eigenvalues is costly and not feasible for large-scale device simulation. We compared the results of the frequently applied triangular well closed-boundary approximation $\mathcal{E}_{\nu, i}=$ $-z_{i}\left(\hbar^{2} / 2 m_{\nu}\right)^{1 / 3} E^{2 / 3}$ with $z_{i}$ being the zeros of the Airy function and $E$ the electric field to the open-boundary eigenvalues as shown in Fig. 5. If the closed-boundary eigenvalues are corrected by an empirical bias-dependent fit function $f\left(V_{\mathrm{GB}}\right)=\alpha-\beta V_{\mathrm{GB}}$ with $\alpha=0.93$ and $\beta=0.03 \mathrm{~V}^{-1}$ for the longitudinal and $\beta=0.05 \mathrm{~V}^{-1}$ for the transversal eigenvalues, very good agreement was achieved.

\section{Results and Conclusions}

The current density applying (2) for a wide range of devices is shown in Fig. 6 and it can be seen that the empirical fit yields excellent agreement to the QBS-based computations. Thus, an easy and stable formula for the evaluation of quantum and continuum tunneling in CMOS devices is achieved, which only poses small additional effort and can be readily implemented in device simulation packages.

\section{References}

[1] W. Magnus and W. Schoenmaker, Microelectronics Reliability 41, 31 (2001).

[2] R. Tsu and L. Esaki, Appl.Phys.Lett. 22, 562 (1973).

[3] Khairurrijal, W. Mizubayashi, S. Miyazaki, and M. Hirose, J.Appl.Phys. 87, 3000 (2000).

[4] E. Anemogiannis, E. N. Glytsis, and T. K. Gaylord, J.Quantum Electronics 29, 2731 (1993).

[5] E. Cassan, J.Appl.Phys. 87, 7931 (2000).

[6] W. R. Frensley, Superlattices \& Microstructures 11, 347 (1992).

[7] R. Clerc, A. Spinelli, G. Ghibaudo, and G. Pananakakis, J.Appl.Phys. 91, 1400 (2002).

[8] A. Gehring, H. Kosina, and S. Selberherr, J.Computational Electronics 2, 219 (2003).

[9] A. Gehring, T. Grasser, H. Kosina, and S. Selberherr, J.Appl.Phys. 92, 6019 (2002).

[10] A. Dalla Serra et al., IEEE Trans.Electron Devices 48, 1811 (2001). 